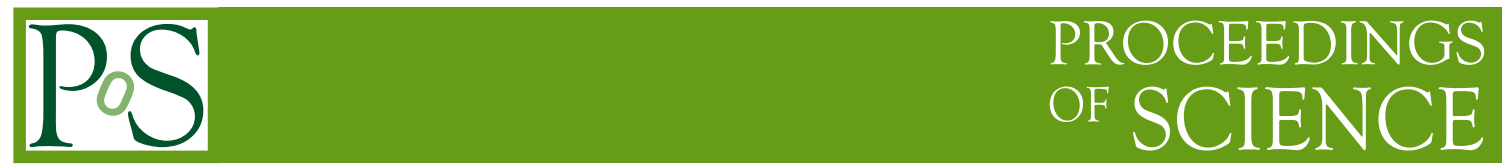

\title{
QCD and forward results from CMS
}

\author{
I. Katkov** \\ Institut für Experimentelle Kernphysik, Karlsruhe Institut für Technologie (KIT), Karlsruhe, \\ Germany \\ E-mail: katkov@kit.edu
}

Highlights of QCD results obtained at the new energy frontier by the CMS experiment based on low pile-up datasets collected in year 2010 are presented. The results rely on excellent performance of tracking as well as unprecedented pseudorapidity coverage achieved with the forward detectors of CMS.

LHC on the March,

November 16-18, 2011

Protvino, Moscow region, Russian Federation

\footnotetext{
*Speaker.

$\dagger$ On behalf of the CMS Collaboration

$\ddagger$ On leave from Skobeltsyn Institute of Nuclear Physics, M.V. Lomonosov Moscow State University.
} 


\section{Motivation}

The clean low pile-up conditions of data taking at LHC in year 2010 enabled to perform thorough tests of QCD, an integral part of the Standard Model. Our hopes for discoveries rest upon such tests.

The LHC is not only the highest energy machine but also the machine where the lowest parton momentum fractions, $x$, can be achieved. The new parton dynamics regime is one of the aspects of the new physics we expect at this energy scale.

Cosmic ray experiments are able to probe even higher energies. Input from the LHC experiments is needed to reduce the uncertainties in determination of the primary energy and composition of the ultra-high energy cosmic-rays.

\section{CMS detector capabilities}

The essential component of CMS is a strong solenoidal magnet operated at $3.8 \mathrm{~T}$ magnetic field. Fully silicon tracking detectors and central calorimeters, crystal electromagnetic and brassscintillator hadronic, are inside the solenoid. The pseudorapidity phase space region in the range below 2.5 units in absolute value covered by the tracking is usually called the central region. Muon chambers of three types are gaseous detectors in the gaps of the return yoke that catches the lines of the magnetic field. For the full detector description see [1].

The CMS is perfectly equipped in the forward region. The region is quite a challenging environment due to the limited space and high radiation levels. Hence CMS has chosen Cherenkov calorimetry for the forward detectors. There are 3 calorimeters of this type: The Hadronic Forward (HF) calorimeter covering the absolute pseudorapidity range from 3 to 5, the CASTOR calorimeter covering the pseudorapidity range from -6.6 to -5.2 , and the Zero Degree Calorimeter (ZDC) that extends the rapidity coverage beyond 8.1 in units of pseudorapidity for neutral particles. Thanks to the TOTEM experiment, tracking is available also in the forward region. The tracking station T1, installed during the 2010-2011 winter technical stop, is in front of the HF and the T2-station is in front of CASTOR. During the same technical stop a set of scintillation counters, Forward Shower Counters (FSC), were mounted around the beam pipe to close the non-instrumented gap between the CASTOR calorimeter and the ZDC. For more details on CMS status and an overview of recent results see [2].

\section{Inelastic proton-proton cross section}

The CMS performed a measurement of a fundamental quantity, the inelastic proton-proton cross section [3]. I am pleased to note that the rise in total hadronic cross-section with energy is a universal phenomenon known as the "Serpukhov effect" for the first time discovered at U70 machine in Protvino. While event pile-up is usually an obstacle, for CMS it is a part of a peculiar method. The method relies mainly on tracking performance. The vertex multiplicity is measured in bins of instantaneous luminosity. A correction is done then accordingly for the vertex reconstruction efficiency for a given minimal number of reconstructed tracks in the central region. Number of vertices depends on the pile-up and hence assuming Poisson dependence of number of 

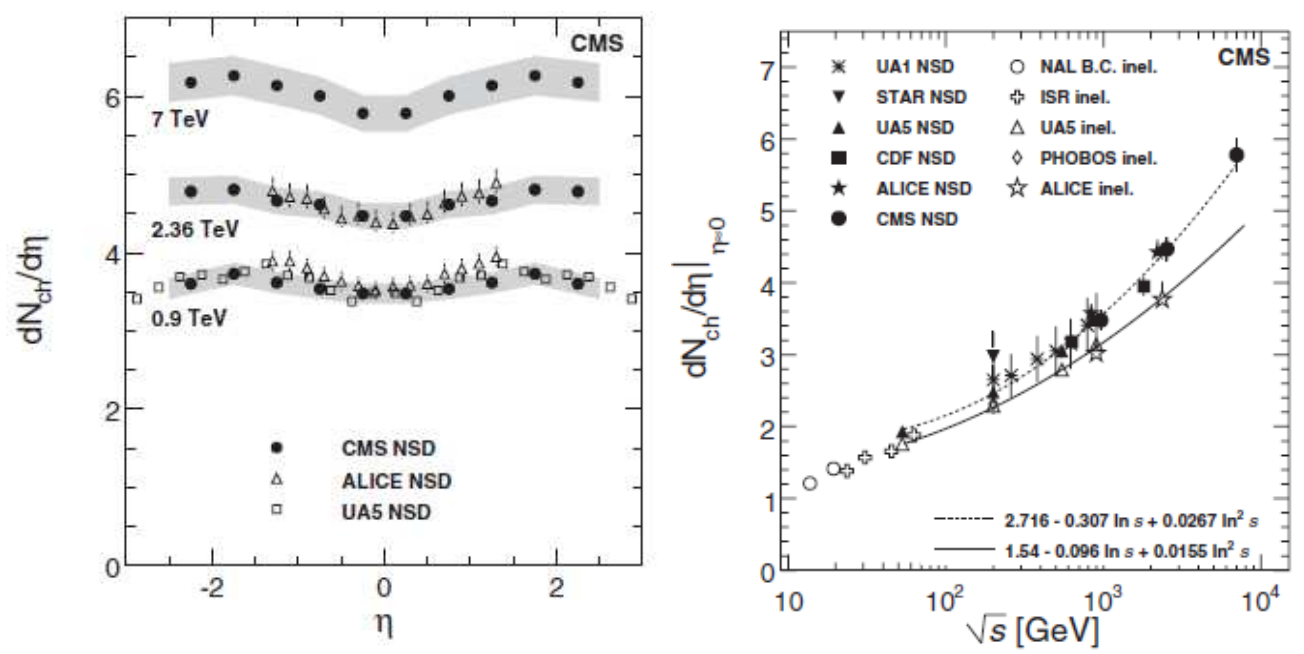

Figure 1: Single charged hadron spectra for NSD events.

pile-up events versus luminosity it is possible to do a fit to extract the visible cross section. The last step is a model dependent extrapolation to the total inelastic cross section. The final value presented along with uncertainties on systematics, luminosity measurement, and extrapolation, is $\sigma_{\text {inel }}=68 \pm 2.0$ (syst.) \pm 2.4 (lumi) \pm 4 (ext.). The result is nicely in line with the result of ATLAS [4] as well as fresh results from the cosmic ray community [5] and the TOTEM experiment [6], shedding also light on the inconsistency observed at TEVATRON energies. A measurement by CMS in the conventional, followed by ATLAS, approach is under way.

\section{Single charged hadron spectra}

The single charged hadron spectra measured by CMS explore the realm of soft QCD at the new energy scale [7]. The data were taken with minimum bias trigger configuration that suppresses single diffractive contributions, so called non-single diffractive data (NSD). Charged hadron pseudorapidity, density at midrapidity and average transverse momentum of hadrons were measured as well as their dependence on the center-of-mass-energies (see Figures 1). Measurements of different experiments are in good agreement. The dependence on the center-of-mass energies is steep, $60 \%$ for the energy rise from $900 \mathrm{GeV}$ to $7 \mathrm{TeV}$. As an example comparison of the data is shown in Figure 2 to a wide spectrum of hadronic models used in cosmic-rays physics [8]. The models embrace the measurements. A reduction of model dependence of the experimental correction factor for the non-single diffractive data was always on the wish list to facilitate comparison to predictions and model tuning. An analysis was performed with a selection reduced to a requirement of 1 track in the central region [9]. Comparison of the data to Pythia tunes indicate that tunes from the pre-LHC era fail at high energies (see Figure 3).

\section{Charged particle multiplicities}

The event-by-event distribution of the charged multiplicity has been measured for NSD-events 


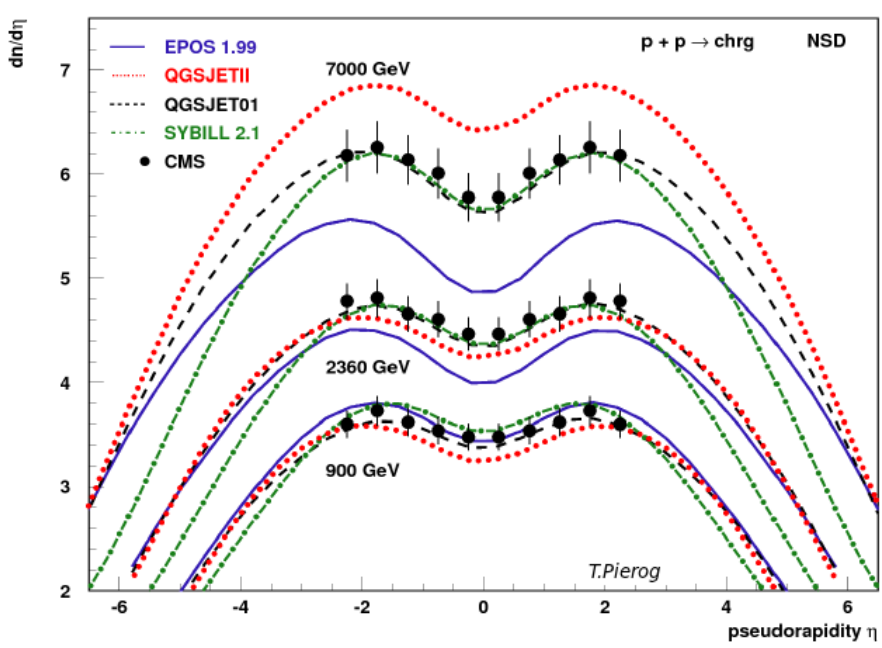

Figure 2: Single charged hadron spectra for NSD events.

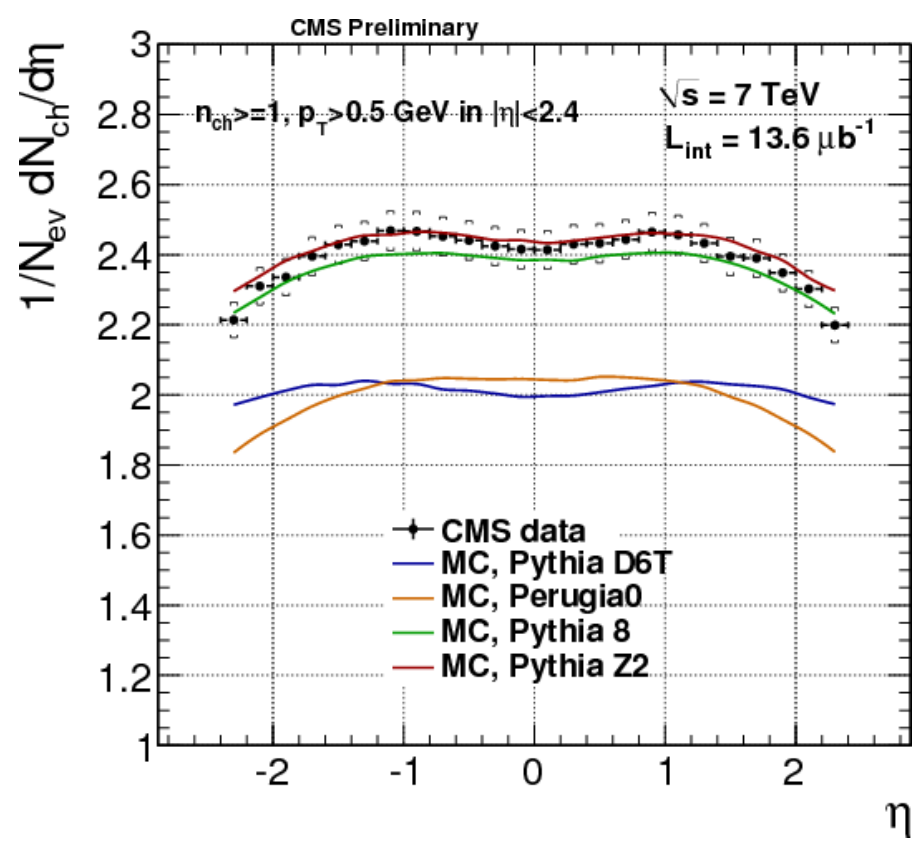

Figure 3: Single charged hadron spectra for events with at least one track.

for 3 centre-of-mass energies [10]. Such measurements provide information on many "tunable" aspects of event modelling such as diffractive production, multi-parton interactions (MPI), saturation effects. The low end of the spectrum is sensitive to diffraction while high multiplicity tail is sensitive to MPI. No model compared to the data can describe properly the energy dependence (see Figure 4). Comparison to the average transverse momentum-versus-multiplicity dependence, showing scaling with the centre-of-mass energy, indicates that no model can describe both quantities at the same time (see Figure 5). 


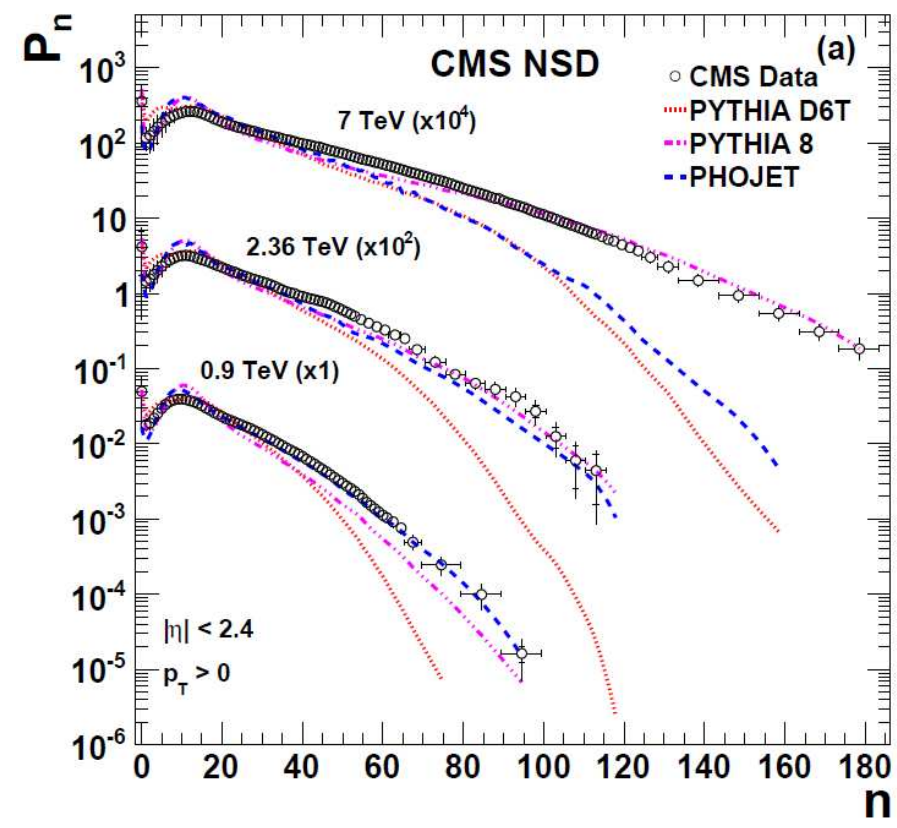

Figure 4: Charged particle multiplicities.

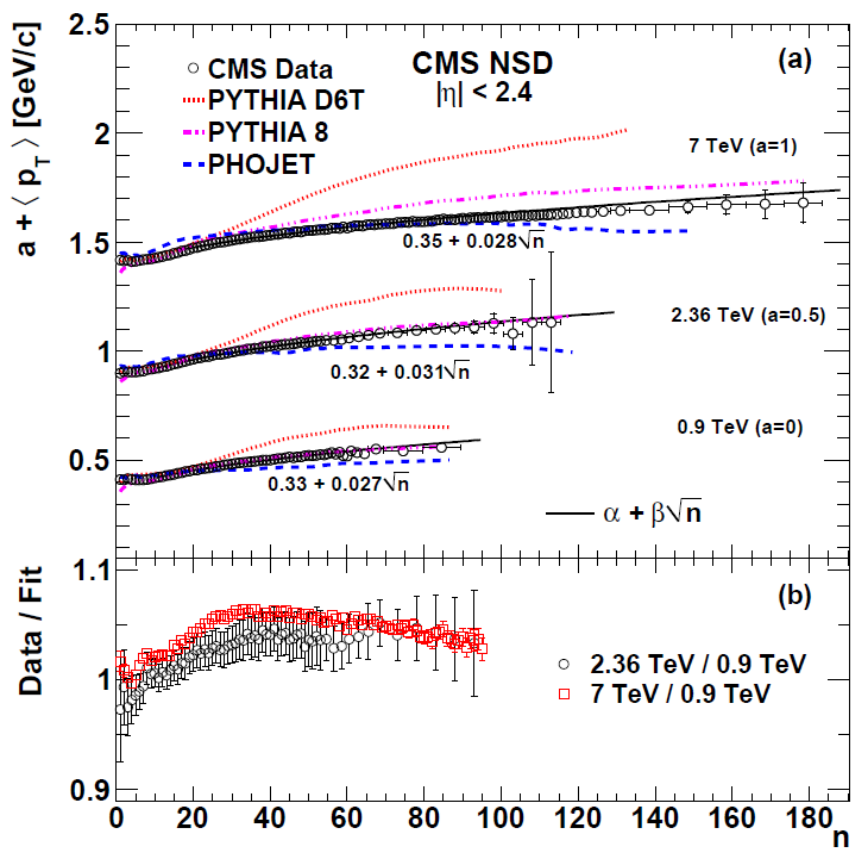

Figure 5: Charged particle multiplicities.

\section{Strange particle production}

A broad range of identified particle spectra measurements is performed by CMS. In particular 
results on the strangeness and baryon production, an important aspect of Monte Carlo modelling, have been published by CMS [11]. Excellent tracker performance allowed of reconstructing complicated cascade decays with good resolution (see Figure 6). Also reconstruction down to very low $p_{t}$ was possible. Pre-LHC Pythia tunes underestimate strange particle production, up to factor of 3 , in particular for high $p_{t}$, for production of hyperons and at high energies (see Figure 7). For more detailed analysis of the topic see [12].

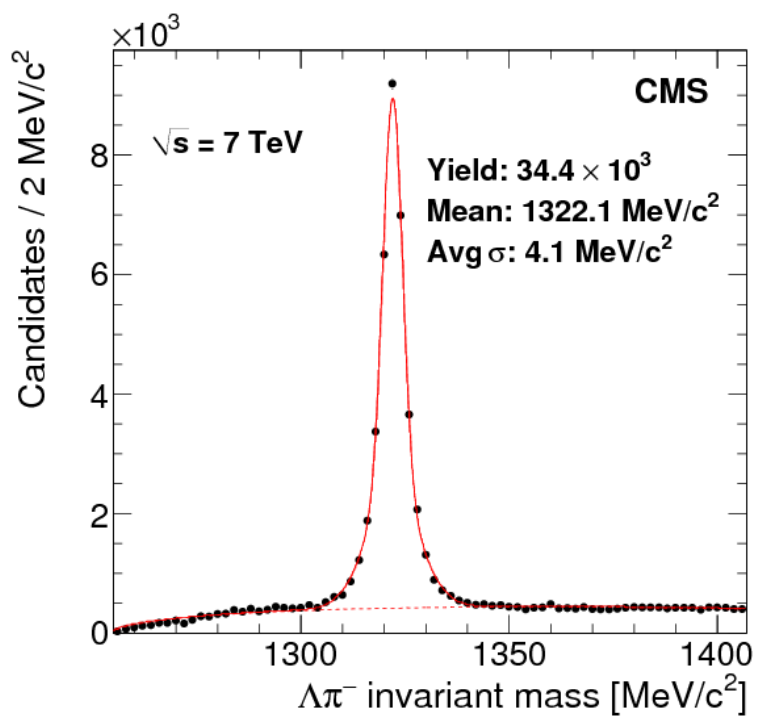

Figure 6: Strange particle production.

\section{Underlying event studies}

CMS has performed classical, a la R. Field [13], measurement of the underlying event activity [14]. Three distinct regions are defined by the dominant momentum flow which is given by the leading track-jet in CMS. Multiplicity and scalar sum of transverse momentum densities are presented in Figure 8 in the transverse region for different energies in comparison to recent Pythia tunes. The recent tunes incorporate also knowledge of LHC results. Strong increase of the UE activity with energy is evident in particular in the ratio plots. The parton shower Monte Carlo tunes can reproduce this behaviour. But this is possible at the costs of worsened consistency with data sets obtained at lower energies.

\section{Measurement of particle correlations}

One of the first significant observations by CMS was the so called "ridge effect" [15]. The idea to look at two-particle correlations in high multiplicity events came from observations made in heavy ion collisions at RHIC. The correlations on $\Delta \eta-\Delta \phi$ plane corresponded to "naive" expectations for usual minimum bias events with such a typical features as jet-related correlations at $\Delta \phi \sim \pi$ and Bose-Einstein correlations at $\Delta \phi$ and $\Delta \eta \sim 0$. The situation changed for events collected with the high multiplicity trigger and for particles in a particular $p_{t}$-range. The long-range 


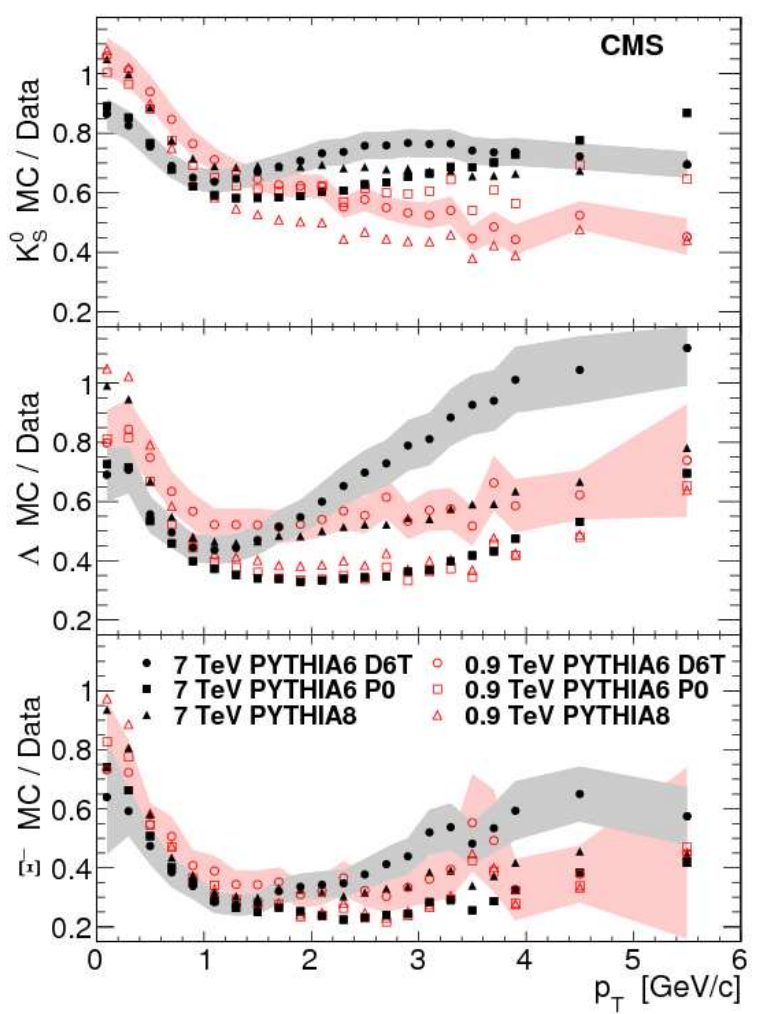

Figure 7: Strange particle production.

$(\Delta \eta)$, near-side $(\Delta \phi)$ correlations were observed (see Figure 9). This is the "ridge" clearly visible at $\Delta \phi \sim 0$. There is no corresponding physics mechanism implemented in the standard Monte Carlo codes. The effect is still under discussion. A collective effect resembling ones observed in heavy ion collisions can be one of possible interpretations.

A dedicated study of the Bose-Einstein correlations have been performed for pairs of samesign charged particles with 4-momentum difference, $Q$, in the range from $0.02 \mathrm{GeV}$ to $2 \mathrm{GeV}$ [16]. From the fit to the region where the correlation, quantified by the double ratio $R=$

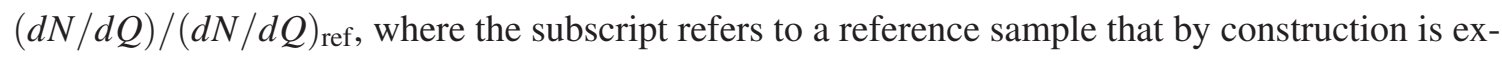
pected to include no effect, is observed one can derive the radius of the effective space-time region emitting bosons with overlapping wave functions. The effective region grows with multiplicity, the dependence on multiplicity also scales with energy (see Figure 10).

\section{Forward energy flow}

So far the potential of the forward instrumentation of CMS was explored in the range up to roughly 5 units in absolute value of pseudorapidity. A energy flow measurement with the forward calorimetry requires a data sample with no pile-up effects. Such kind of studies are directly sensitive to the amount of parton radiation and multi-parton interactions. The CMS published recently results with corrected distributions for the dependence of the energy flow on the rapidity and the center-of-mass energy for minimum bias and dijet events [17]. One observation is a strong rise 

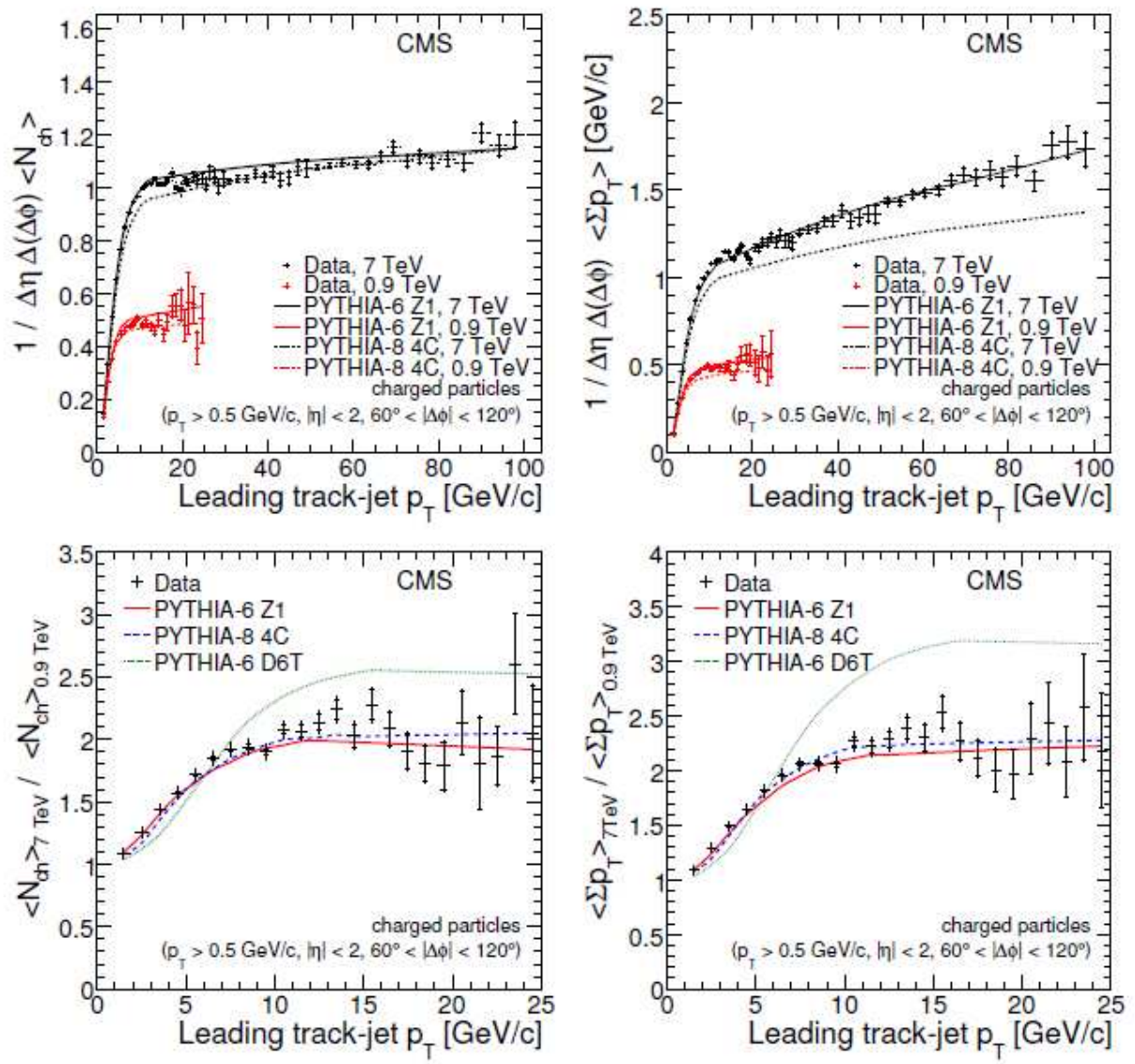

Figure 8: Underlying event activity.

(d) $\mathrm{CMS} \mathrm{N} \geq 110,1.0 \mathrm{GeV} / \mathrm{c}<\mathrm{p}_{\mathrm{T}}<3.0 \mathrm{GeV} / \mathrm{c}$

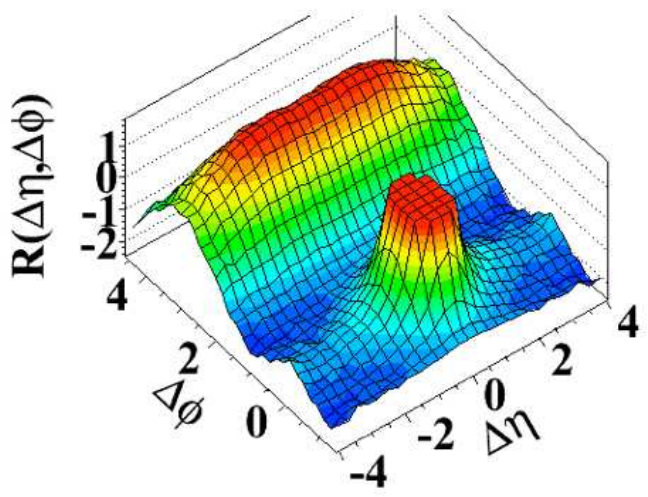

Figure 9: Particle correlations. 


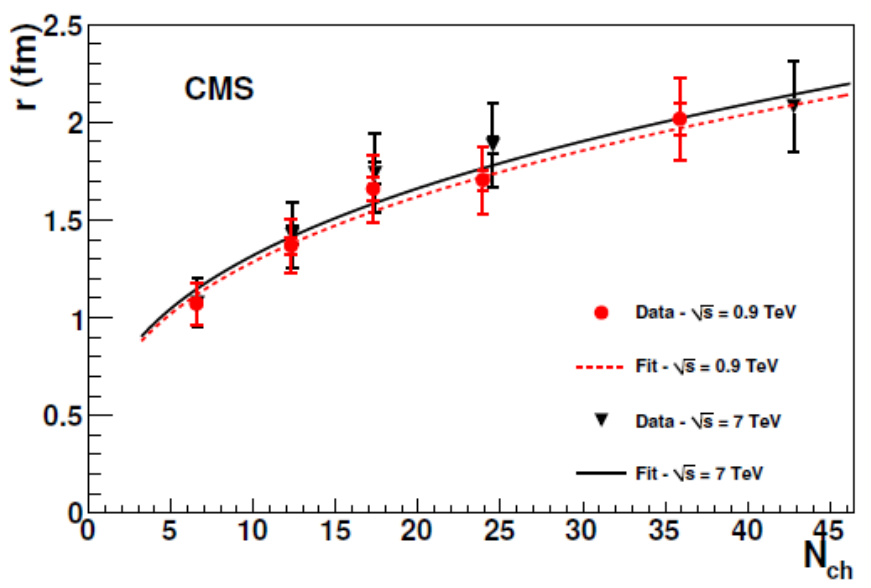

Figure 10: Particle correlations.

of the energy flow with energy. Also the "pedestal effect" is observed, namely the energy flow increases in a sub-sample of events with two jets in the central region. Models without MPI fail to describe the data. Hadronic models used in cosmic-rays physics embrace the measurements and provide the best description as shown in Figure 11.
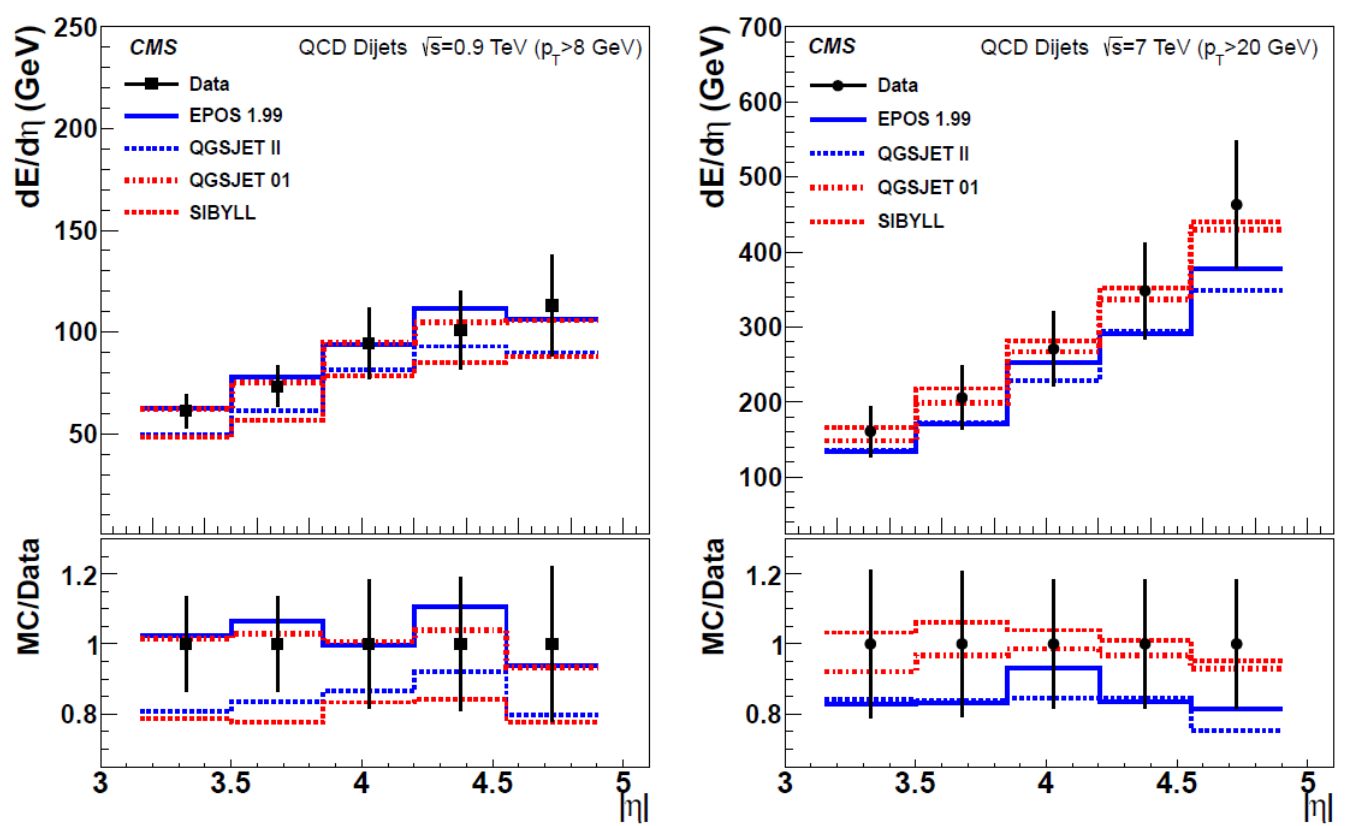

Figure 11: Forward energy flow.

\section{Jets in the central and forward regions}

The domain of hard QCD was extensively explored by CMS. Inclusive jet cross sections dou- 
ble differential in $p_{t}$ and rapidity have been measured using the anti- $k_{t}$ particle flow jets with the cone radius of 0.5 [18]. For the central rapidities up to 3 units the measurement covers a very wide range in $p_{t}$ from $18 \mathrm{GeV}$ to $1.1 \mathrm{TeV}$. The results are compared to the Next-to-Leading-Order calculations. Work is ongoing to extend the $p_{t}$ coverage even further and on improving the correction procedure.

The ratio of the data to the predictions is shown for different parton distribution function used in the theoretical calculations along with corresponding uncertainties [19]. This is shown in Figure 12 for two extreme central bins. Better agreement is observed in more central bins and at higher $p_{t}$.
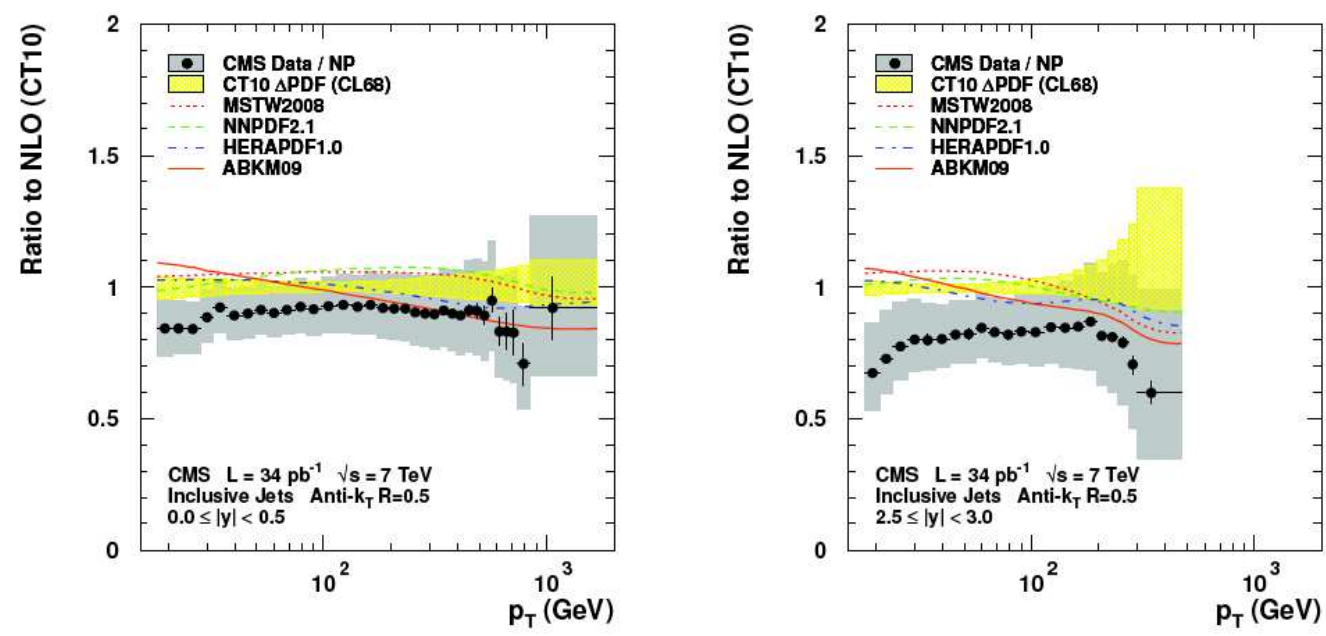

Figure 12: Inclusive jet measurements compared to NLO PDFs.

Similar measurement and comparison has been done for forward rapidities in the acceptance of the HF calorimeter [20]. It is interesting to note that such a measurement is sensitive to the parton distributions both at low- and high- $x$ values. The agreement with the wide variety of predictions is reasonable within large energy scale uncertainties in the data. Work is ongoing to improve the situation with the energy scale using in situ calibration procedures. Forward jets attract in particular interest in the context of exclusive Higgs boson searches. Forward-central jet correlations are expected to reveal enhanced sensitivity to effects of parton dynamics in the new kinematic regime. The measured forward-central jet spectra are double differential in transverse momentum and pseudorapidity. Comparison in Figure 13 is done to Pythia tunes, NLO matrix elements interfaced to parton showering (POWHEG), and CASCADE Monte Carlo implementing CCFM evolution in the initial parton cascade. A wide spread is observed in the predictions. Even taking into account large energy scale uncertainties, such kind of correlations pose a challenge for theoretical description.

\section{Energy flow with $W / Z$ production}

One more option to explore the underlying event, MPI, and hard diffraction was used by CMS reconstructing $W$ and $Z$ boson in the central region and at the same time observing the forward energy flow [21]. The spread in predictions is large but a reasonable description of the energy flow 


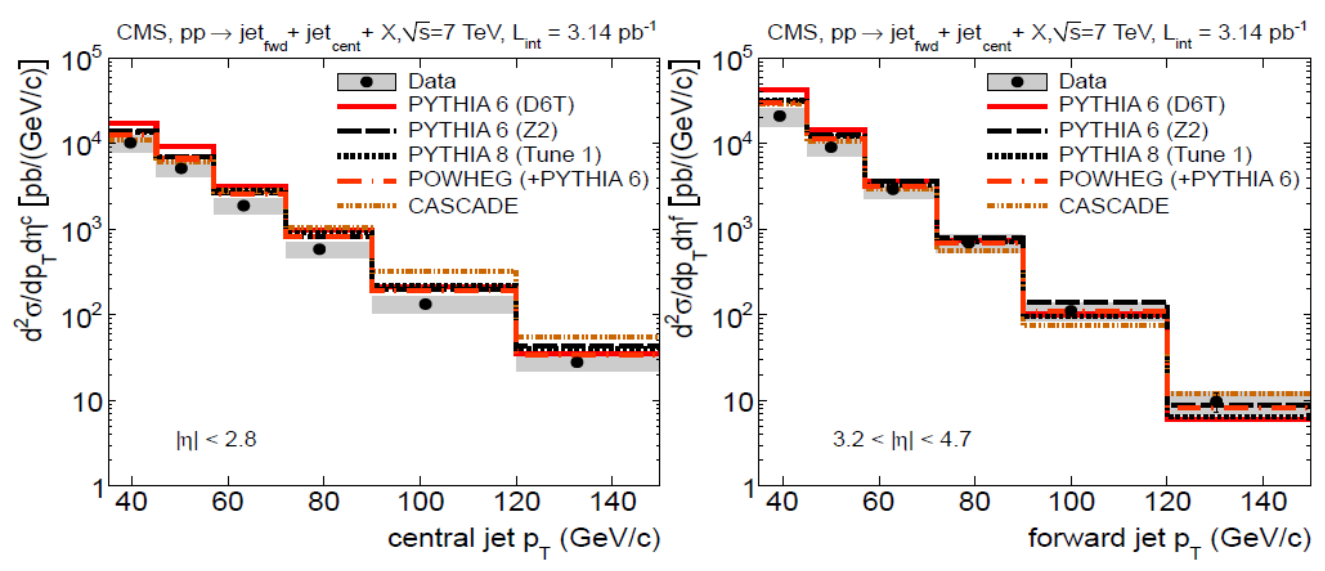

Figure 13: Differential distributions for central-forward jets.

is possible. Strong forward-backward correlations, observed in events with reconstructed $W$ and $Z$ bosons, are not well modelled.

\section{Inclusive to exclusive dijet ratio}

The effects beyond the DGLAP evolution were searched for in the analysis of dijet events with as large rapidity separation, $\Delta y$, of the jets as possible [22]. The ratio, $R=\sigma_{\text {incl }} / \sigma_{\text {excl }}$, also called " $k_{t}$-factor", is expected to rise with $\Delta y$ as phase space opens for parton emissions. Interesting enough, the observed rise was found to be in agreement with PYTHIA modelling within the energy scale uncertainties (see Figure 14).

\section{Studies with dijets and diphotons}

Measurements with dijets and diphotons provide a well-known testbed for the perturbative QCD. Studies of angular and invariant mass distribution of dijets in the central region have been performed $[23,24]$. Low values of $\chi$, a practical replacement of the polar angle, $\theta^{*}$, and high invariant masses, $m_{j j}$, is the region where new phenomena like contact interactions are expected (see Figure 15). Dijet azimuthal decorrelations can be well described with perturbative QCD at NLO calculations as well as with parton shower Monte Carlo models. Comparison to models reveals sensitivity to the modelling of the initial state radiation.

Production of pairs of isolated photons in QCD is an irreducible background to the searches of the Higgs boson in the diphoton decay mode. An excess was observed by CMS [25] at low $\Delta \phi_{\gamma \gamma}$, the azimuthal separation of the photons, compared to a NLO QCD prediction (see Figures 16-17). The effect is argued to be due to higher-order QCD terms not accounted for in the NLO prediction.

\section{Summary}

A wide spectrum of QCD measurements has been performed by CMS at LHC energies which 


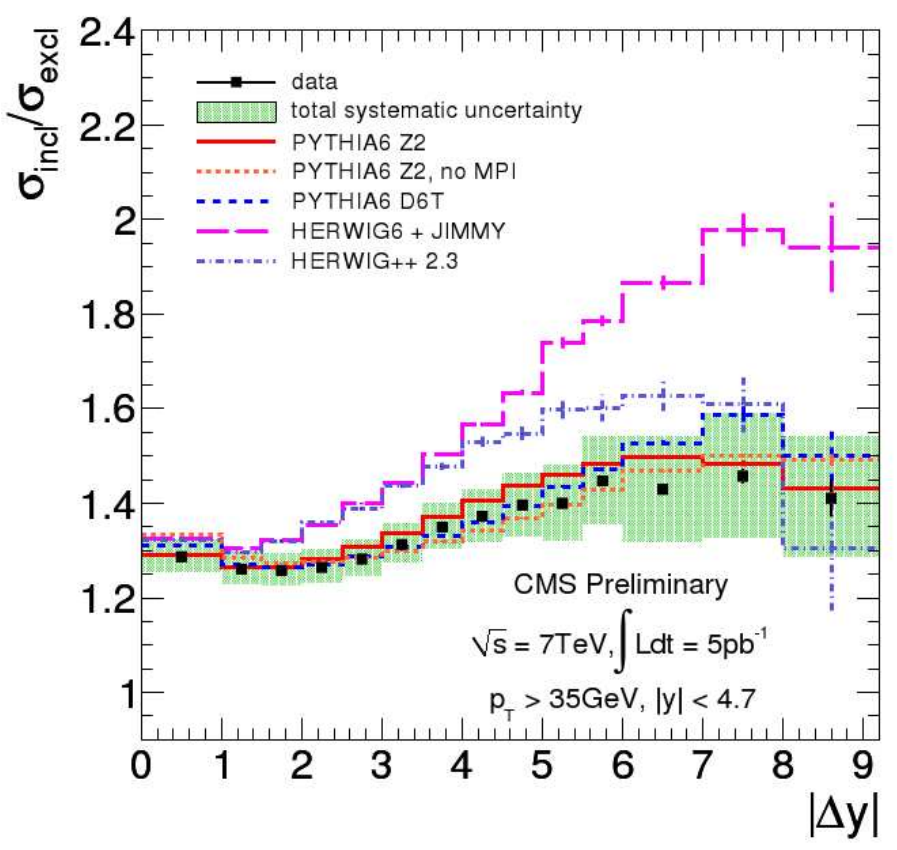

Figure 14: Dijet differential distributions.

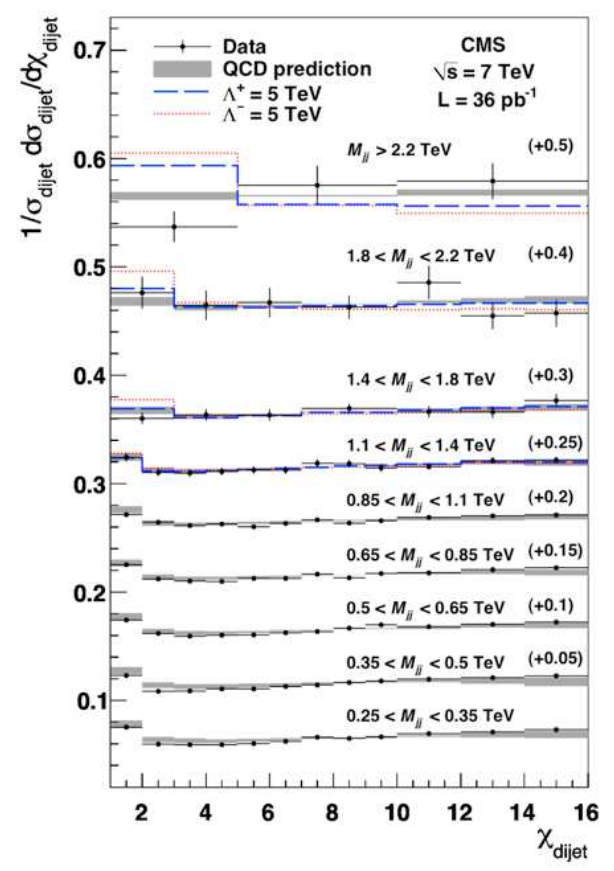

Figure 15: Dijet differential distributions. 


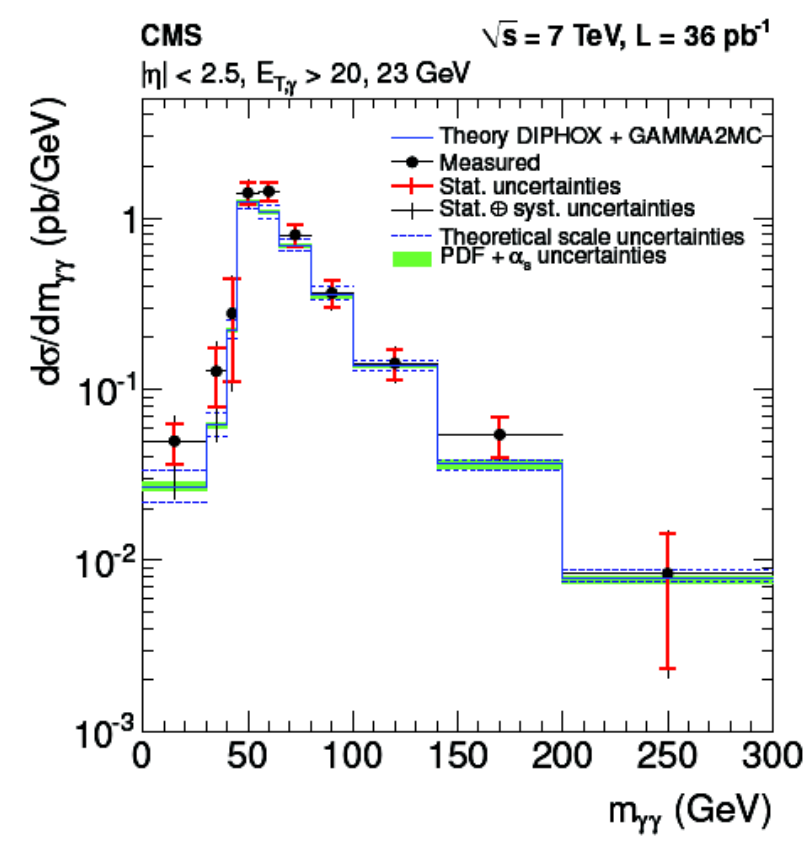

Figure 16: Diphoton differential distributions.

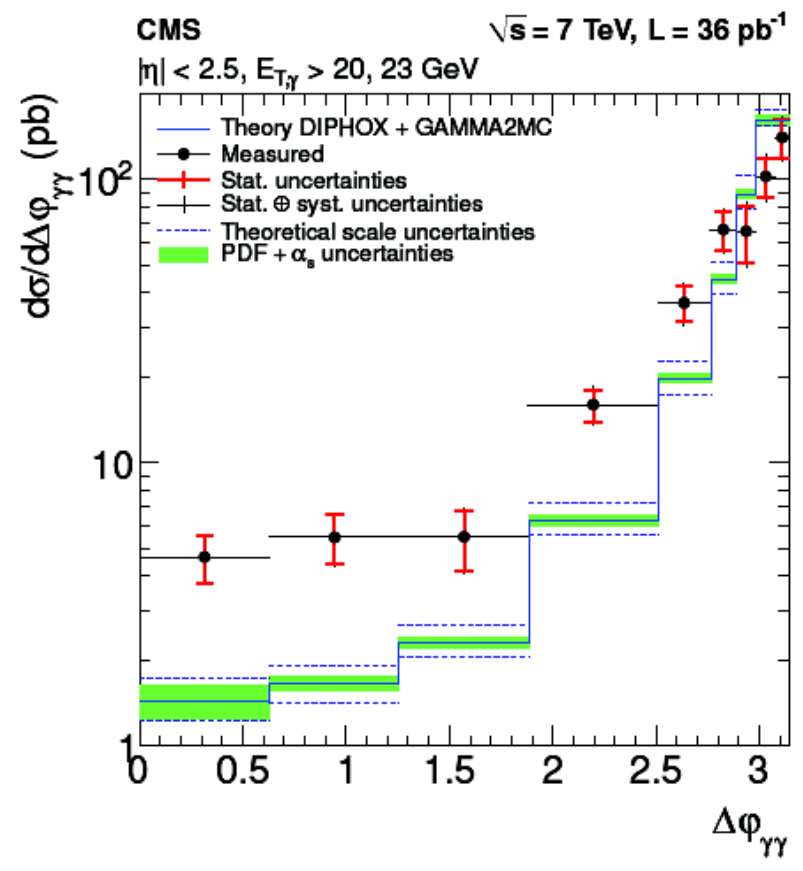

Figure 17: Diphoton differential distributions.

will contribute to the overall better understanding of underlying physics and better Monte Carlo modelling.

Fully inclusive measurements are reasonably described by the predictions presented here. At 
the same time in many cases measurements of correlations pose a real challenge.

Especially high- $p_{t}$ studies can profit from huge data sample of year 2011. Discussions are under way on a possibility for soft QCD and forward analyses to extend their data samples in dedicated LHC low luminosity runs.

\section{Acknowledgments}

Efforts of physicists, engineers and technicians contributed to the construction, operation and support of the LHC and the CMS experiment are invaluable. This work was supported by the Helmholtz-Nachwuchsgruppen grant VH-NG-733.

\section{References}

[1] CMS Collab., The CMS experiment at the CERN LHC, JINST 3, S08004 (2008).

[2] M. Jeitler, Status and Performance of CMS, this volume.

[3] CMS Collab., Measurement of the inelastic pp cross section at $\sqrt{s}=7 \mathrm{TeV}$ with the CMS detector, CMS-PAS-FWD-11-001, CERN, Geneva, 2011.

[4] ATLAS Collab., Measurement of the inelastic proton-proton cross-section at $\sqrt{s}=7 \mathrm{TeV}$ with the ATLAS detector, Nature Commun. 2, 463 (2011).

[5] R. Ulrich for the Pierre Auger Collab., Estimate of the proton-air cross section with the Pierre Auger Observatory, 32nd International Cosmic Ray Conference, Beijing 2011, arXiv:1107.4804 [astro-phys.HE].

[6] TOTEM Collab., First measurement of the total proton-proton cross section at the LHC energy of $\sqrt{s}=7 \mathrm{TeV}$, Euro Phys. Lett. 96, 21002 (2011).

[7] CMS Collab., Transverse-momentum and pseudorapidity distributions of charged hadrons in pp collisions at $\sqrt{s}=7 \mathrm{TeV}$, Phys. Rev. Lett. 105, 022002 (2010).

[8] D. d'Enterria, R. Engel, T. Pierog, S. Ostapchenko and K. Werner, Constraints from the first LHC data on hadronic event generators for ultra-high energy cosmic-ray physics, Astropart. Phys. 35, 98 (2011).

[9] CMS Collab., Pseudorapidity distributions of charged particles in pp collisions at $\sqrt{s}=7 \mathrm{TeV}$ with at least one central charged particles, CMS-PAPER-QCD-10-024, CERN, Geneva, 2010.

[10] CMS Collab., JHEP 01, 079 (2011).

[11] CMS Collab., Strange particle production in pp collisions at $\sqrt{s}=0.9$ and $7 \mathrm{TeV}$, JHEP 05, 064 (2011)

[12] L. Smirnova, $K / \pi$ ratio and strangeness suppression in pp collisions at the $L H C$, this volume.

[13] CDF Collab. (R. Field), Studying the 'underlying event' at CDF and the LHC,

FERMILAB-CONF-09-792-E, Fermilab, 2009.

[14] CMS Collab., Measurement of the underlying event activity at the LHC with $\sqrt{s}=7 \mathrm{TeV}$ and comparison with $\sqrt{s}=0.9 \mathrm{TeV}$, JHEP 09, 109 (2011).

[15] CMS Collab., Observation of Long-Range Near-Side Angular Correlations in Proton-Proton Collisions at the LHC, JHEP 09, 091 (2010). 
[16] CMS Collab., Measurement of Bose-Einstein Correlations in pp Collisions at $\sqrt{s}=0.9$ and $7 \mathrm{TeV}$, JHEP 05, 029 (2011).

[17] CMS Collab., Measurement of energy flow at large pseudorapidities in pp collisions at $\sqrt{s}=0.9$ and $7 \mathrm{TeV}$, JHEP 11, 148 (2011).

[18] CMS Collab., Measurement of the Inclusive Jet Cross Section in pp Collisions at $\sqrt{s}=7$ TeV, Phys. Rev. Lett. 107, 132001 (2011).

[19] CMS Collab., Comparison of Inclusive Jet and Dijet Mass Cross Sections at $\sqrt{s}=7$ TeV with Predictions of perturbative QCD, CMS-NOTE-2011-004, CERN, Geneva, 2011.

[20] CMS Collab., Measurement of the inclusive production cross sections for forward jets and for dijet events with one forward and one central jet in pp collisions at $\sqrt{s}=7 \mathrm{TeV}$, arXiv:1202.0704 [hep-ex].

[21] CMS Collab., Forward Energy Flow, Central Charged-Particle Multiplicities, and Pseudorapidity Gaps in $W$ and $Z$ Boson Events from pp Collisions at $7 \mathrm{TeV}$, arXiv:1110.0181 [hep-ex].

[22] CMS Collab., Measurement of inclusive and exclusive dijet production ratio at large rapidity intervals at $\sqrt{s}=7 \mathrm{TeV}$, CMS-PAS-FWD-10-014, CERN, Geneva, 2011.

[23] CMS Collab., Measurement of Dijet Angular Distributions and Search for Quark Compositeness in pp Collisions at 7 TeV, Phys. Rev. Lett. 106, 201804 (2011).

[24] CMS Collab., Dijet Azimuthal Decorrelations in pp Collisions at $\sqrt{s}=7 T e V$, Phys. Rev. Lett. 106, 122003 (2011).

[25] CMS Collab., Measurement of the production cross section for pairs of isolated photons in pp collisions at $\sqrt{s}=7 \mathrm{TeV}$, JHEP 01, 133 (2012). 